\title{
ON THE ASYMPTOTIC NUMBER OF EDGE STATES FOR MAGNETIC SCHRÖDINGER OPERATORS
}

\author{
RUPERT L. FRANK
}

\begin{abstract}
We consider a Schrödinger operator $(h \mathbf{D}-\mathbf{A})^{2}$ with a positive magnetic field $B=\operatorname{curl} \mathbf{A}$ in a domain $\Omega \subset \mathbb{R}^{2}$. The imposing of Neumann boundary conditions leads to spectrum below $h \inf B$. This is a boundary effect and it is related to the existence of edge states of the system.

We show that the number of these eigenvalues, in the semi-classical limit $h \rightarrow 0$, is governed by a Weyl-type law and that it involves a symbol on $\partial \Omega$. In the particular case of a constant magnetic field, the curvature plays a major role.
\end{abstract}

\section{INTRODUCTION AND MAIN RESUltS}

1.1. Introduction. In this paper we consider a magnetic Schrödinger operator

$$
P_{h}:=(h \mathbf{D}-\mathbf{A})^{2} \quad \text { in } L_{2}(\Omega)
$$

where $\mathbf{D}=-i \nabla$ and $\Omega \subset \mathbb{R}^{2}$ has a smooth and compact boundary $\partial \Omega$ on which we impose Neumann boundary conditions. The 'magnetic field' $\operatorname{curl} \mathbf{A}=B$ is assumed to be smooth, positive and 'not too large on the boundary' (see (1.6) below for the precise assumption). As we will see, the choice of Neumann boundary conditions implies the existence of eigenvalues below $h \inf B$. Our goal is to determine for $b_{0}<\inf B$ the asymptotic behavior of the number

$$
N\left(h b_{0}, P_{h}\right)
$$

of eigenvalues of $P_{h}$ below $h b_{0}$ in the semi-classical limit $h \rightarrow 0$. The semiclassical limit is of course equivalent to the limit of a strong magnetic field.

The operator (1.1) has recently received a lot of attention in connection with the Ginzburg-Landau theory of superconductivity. Indeed, the lowest eigenvalue of (1.1) plays an important role in the description of the boundary nucleation of superconductity close to the third critical field, see [LuPa], HeMo, FoHe2 and references therein. The methods developed in these papers will also be important for our analysis. They show in particular that the boundary has an effect similar to that of a potential well and that eigenfunctions of $P_{h}$ corresponding to eigenvalues below $h$ inf $B$ are strongly localized near the boundary. Hence they correspond to 'edge states'.

Date: February 7, 2008. 
We emphasize that the 'energy level' in (1.2) depends on $h$. If instead the energy $\lambda>0$ is fixed, it is well-known that

$$
N\left(\lambda, P_{h}\right) \sim h^{-2} \lambda \frac{|\Omega|}{4 \pi}
$$

for bounded $\Omega$. In particular, the leading term is independent of the magnetic field. Recalling that the distance between two Landau levels is proportional to $h$ it is natural to expect effects of the magnetic field to appear when $\lambda=b_{0} h$. We note also that (1.2) can be rewritten as

$$
N\left(h b_{0}, P_{h}\right)=N\left(b_{0},\left(h^{1 / 2} \mathbf{D}-h^{-1 / 2} \mathbf{A}\right)^{2}\right) .
$$

This suggests that the effective semi-classical parameter of the problem is $h^{1 / 2}$, which tends to zero simultaneously as the effective magnetic field $h^{-1 / 2} B$ tends to infinity.

The first results on spectral asymptotics of the operator (1.1) were obtained by Colin de Verdière [CV] and Tamura [Ta]. The former author considers a slightly different problem, namely the behavior of $N\left(\lambda, P_{1}\right)$ as $\lambda \rightarrow \infty$. Moreover, $P_{1}$ is defined there as the Dirichlet realization of (1.1). The methods of $[\mathrm{CV}]$ (see also $[\mathrm{Tr}$ ) allow to prove that under suitable assumptions on $\mathbf{A}$ one has for all $b_{0}>0$

$$
N\left(h b_{0}, P_{h}\right) \sim h^{-1} \frac{1}{2 \pi} \sum_{n=1}^{\infty} \int_{\left\{x \in \Omega:(2 n-1) B(x)<b_{0}\right\}} B(x) d x .
$$

This formula is valid both for the Dirichlet and for the Neumann realization. However, while the Dirichlet realization has no spectrum below $h \inf B$, this is no longer true for the Neumann realization. In this case (1.3) provides the estimate

$$
N\left(h b_{0}, P_{h}\right)=o\left(h^{-1}\right), \quad b_{0} \leq \inf B .
$$

Our goal is to improve upon this and to determine the precise behavior of $N\left(h b_{0}, P_{h}\right)$ as $h \rightarrow 0$. So loosely speaking we are interested in the leading term of the spectral asymptotics whose first term vanishes.

For further results on spectral asymptotics of magnetic Schrödinger operators we refer to [LiSoYn, IV], DiRa] and references therein.

1.2. Main result. Let us state the precise assumptions on $\Omega$ and $\mathbf{A}$ under which we shall work henceforth. We assume that $\Omega \subset \mathbb{R}^{2}$ is an open domain with $\partial \Omega \in C^{4}$. Moreover, for the sake of simplicity, we assume that $\partial \Omega$ is bounded and connected. Note that we do not assume that $\Omega$ is bounded or simply connected. We consider a 'magnetic vector potential' $\mathbf{A} \in C^{2}\left(\bar{\Omega}, \mathbb{R}^{2}\right)$ and introduce the 'magnetic field' $B:=\operatorname{curl} \mathbf{A}$ and the quantities

$$
b:=\inf _{x \in \Omega} B(x), \quad b^{\prime}:=\inf _{x \in \partial \Omega} B(x) .
$$

The operator (1.1) is defined via the quadratic form

$$
q_{h}[u]:=\int_{\Omega}|(h \mathbf{D}-\mathbf{A}) u|^{2} d x
$$


with domain

$$
\mathcal{D}\left[q_{h}\right]:=\left\{u \in L_{2}(\Omega):(h \mathbf{D}-\mathbf{A}) u \in L_{2}\left(\Omega, \mathbb{C}^{2}\right)\right\} .
$$

If the essential spectrum of $P_{h}$ is non-empty, which may happen if $\Omega$ is unbounded, we use the well-known inequality

$$
h b \int_{\Omega}|u|^{2} d x \leq \int_{\Omega}|(h \mathbf{D}-\mathbf{A}) u|^{2} d x, \quad u \in C_{0}^{\infty}(\Omega),
$$

and a 'magnetic version' of Persson's lemma to conclude that inf $\sigma_{e s s}\left(P_{h}\right) \geq$ $h b$. Hence in any case for $\lambda<h b$ the spectrum of $P_{h}$ in the interval $[0, \lambda)$ consists of finitely many eigenvalues of finite multiplicities, and we denote their total number (taking multiplicities into account) by $N\left(\lambda, P_{h}\right)$.

In order to state our main results we need some notation. For $\xi \in \mathbb{R}$ we denote by $\mu(\xi)$ the lowest eigenvalue of the operator

$$
-\frac{d^{2}}{d t^{2}}+(\xi+t)^{2} \quad \text { in } L_{2}\left(\mathbb{R}_{+}\right)
$$

with a Neumann boundary condition at the origin. Then (see Subsection 2.1 for more details) the minimum

$$
\Theta_{0}:=\inf _{\xi \in \mathbb{R}} \mu(\xi)
$$

is attained at a unique $\xi_{0}$ and one has $\xi_{0} \in(-1,0)$ and

$$
\mathcal{C}_{1}:=\mu^{\prime \prime}\left(\xi_{0}\right) / 6\left|\xi_{0}\right|>0 \text {. }
$$

Throughout the following we shall assume that

$$
0<\Theta_{0} b^{\prime}<b .
$$

Since $0<\Theta_{0}<1$ (numerically $\Theta_{0}=0.59 \ldots$ ) this is in particular true in the important special case of a constant magnetic field.

Our first main result is the following.

Theorem 1.1. Let $\Theta_{0} b^{\prime}<b_{0}<b$. Then

$$
\lim _{h \rightarrow 0} h^{1 / 2} N\left(h b_{0}, P_{h}\right)=\frac{1}{2 \pi} \iint_{\left\{(x, \xi) \in \partial \Omega \times \mathbb{R}: B(x) \mu(\xi)<b_{0}\right\}} B(x)^{1 / 2} d s(x) d \xi
$$

We emphasize that (1.7) has a Weyl-type form, involving the symbol $B(x) \mu(\xi)$ on the co-tangent bundle of the one-dimensional manifold $\partial \Omega$. The essentially one-dimensional nature of the asymptotics is also reflected in the fact that the effective semi-classical parameter $h^{1 / 2}$ appears with the power -1 in the asymptotics. This should be compared with (1.3) for $b_{0}>b$. There both the integral term and the power of $h^{1 / 2}$ reflect the two-dimensional nature of the bulk states.

Note that Theorem 1.1 implies that $N\left(h \Theta_{0} b^{\prime}, P_{h}\right)=o\left(h^{-1 / 2}\right)$. It is natural to ask whether it is possible to obtain the correct asymptotics. This will probably involve the geometry of the set $\left\{x \in \partial \Omega: B(x)=b^{\prime}\right\}$. Here we give an answer in the particular case where the magnetic field $B$ is constant. Indeed, we do not only give the asymptotics of $N\left(h b_{0}, P_{h}\right)$ for $b_{0}=\Theta_{0} B$ 
but we allow $b_{0}$ to vary with $h$ on the scale $h^{1 / 2}$. The result will involve the curvature $\kappa: \mathbb{R} /|\partial \Omega| \mathbb{Z} \rightarrow \mathbb{R}$, see Subsection 2.2 for our notation. We follow the usual convention that $\kappa \geq 0$ if $\Omega$ is convex. We will prove

Theorem 1.2. Assume that $B$ is constant and let $\kappa_{0} \in \mathbb{R}$. Then

$$
\lim _{h \rightarrow 0} h^{1 / 4} N\left(h \Theta_{0} B+h^{3 / 2} \mathcal{C}_{1} B^{1 / 2} \kappa_{0}, P_{h}\right)=\frac{B^{1 / 4}}{\pi \sqrt{3\left|\xi_{0}\right|}} \int_{0}^{|\partial \Omega|}\left(\kappa(s)+\kappa_{0}\right)_{+}^{1 / 2} d s .
$$

There is an important difference between Theorems 1.1 and 1.2. If $\mathcal{O}$ is a bounded domain we can consider both the interior problem $\Omega=\mathcal{O}$ and the exterior problem $\Omega=\mathbb{R}^{2} \backslash \mathcal{O}$. Now if $b_{0}>\Theta_{0} b^{\prime}$ it follows from (1.7) that the leading order terms of $N\left(h b_{0}, P_{h}\right)$ for both problems coincide (provided the magnetic fields coincide on the boundary). This is no longer true if $B$ is constant and $b_{0}=\Theta_{0} B$. Indeed, the asymptotics are 'complementary' in the following sense: for the interior problem $N\left(h \Theta_{0} B, P_{h}\right)$ is, up to leading order, determined by the convex part of the boundary (where the curvature of $\mathcal{O}$ is positive) and for the exterior problem $N\left(h \Theta_{0} B, P_{h}\right)$ is determined by the concave part (where the curvature of the obstacle $\mathcal{O}$ is negative). This observation is in the same spirit as the considerations on spectral duality in HoSm.

Remark 1.3. Let us mention an immediate generalization of Theorems 1.1 1.2 and their proofs. If $\partial \Omega$ has finitely many connected components and if one imposes on each of them either Dirichlet or Neumann conditions, then a formula similar to (1.7) holds, but the integration is restricted to the Neumann components. Moreover, it is enough that only the boundary of the Neumann components is $C^{4}$, and also that the assumption $\mathbf{A} \in C^{2}$ holds only in a neighborhood of those components.

Moreover, it would be desirable to remove the assumption of smoothness of the boundary. If $\partial \Omega$ is piecewise smooth one can probably use the methods from Bon.

1.3. Outline of the paper. The proofs of Theorems 1.1 and 1.2 are technical but the main idea is rather simple. To show (1.7) we localize the problem, following $[\mathrm{HeMo}$, to a tubular neighborhood of the boundary of normal size $h^{3 / 8}$ and cut this into boxes of tangential size $h^{3 / 8}$. In each of these boxes we approximate the magnetic field by a constant one, see Subsection 3.3. This reduces the problem to the analysis of the model problem of an operator with constant field in a rectangle with Neumann boundary conditions on one edge and Dirichlet boundary conditions elsewhere. For this operator we cannot separate variables but it turns out (Subsection 3.2) that its spectral counting function is fairly close to the one of the operator on an infinite half-cylinder. The latter problem is treated in Subsection 3.1. The reduction to the model problem and a careful estimate of the remainder is achieved in Subsection 3.4. In Subsection 3.5 we complete the proof of Theorem 1.1. We will even obtain a remainder estimate. 
The proof of Theorem 1.2 follows a similar pattern and we will be rather succinct there. The analysis of the model problem is, however, significantly more difficult, see Subsection 4.1 .

On a technical level we mention that classical Dirichlet-Neumann bracketing is not possible in our situation, since additional Neumann boundary conditions would produce too many additional eigenvalues. This difficulty is overcome in CV by a localization technique based on the IMS formula. We emphasize once more that the paper [CV] concerns Dirichlet boundary conditions, so that the boundary effects of our Theorems 1.1 and 1.2 were not present there.

1.4. Acknowledgements. The author wishes to thank Prof. B. Helffer for the invitation to Orsay and numerous fruitful discussions. He is also grateful to S. Fournais and A. Hansson for useful remarks. Financial support through the ESF Scientific Programme in Spectral Theory and Partial Differential Equations (SPECT) as well as through the European Research Network "Postdoctoral Training Program in Mathematical Analysis of Large Quantum Systems" (Contract Number HPRN-CT-2002-00277) is gratefully acknowledged.

\section{Auxiliary material}

2.1. A family of ordinary differential operators. For any $\xi \in \mathbb{R}$ we consider the operator

$$
-\frac{d^{2}}{d t^{2}}+(\xi+t)^{2} \quad \text { in } L_{2}\left(\mathbb{R}_{+}\right)
$$

with a Neumann boundary condition at the origin and denote its lowest eigenvalue by $\mu(\xi)$. (Here we use the convention of [FoHe1]; we note that in HeMo $\xi$ is replaced by $-\xi$.)

The dependence of $\mu(\xi)$ on $\xi$ has been studied in [DaHe (see also [Bol]) where the following facts were established: The function $\mu$ is smooth and satisfies $\lim _{\xi \rightarrow-\infty} \mu(\xi)=1, \lim _{\xi \rightarrow+\infty} \mu(\xi)=+\infty$. There exists a $\xi_{0} \in$ $(-1,0)$ such that $\mu$ is strictly decreasing in $\left(-\infty, \xi_{0}\right)$ and strictly increasing in $\left(\xi_{0}, \infty\right)$. Moreover, at $\xi_{0}$ it has a non-degenerate minimum and one has

$$
\Theta_{0}:=\mu\left(\xi_{0}\right)=\xi_{0}^{2}
$$

In view of these facts it is possible to introduce two inverse functions $\nu_{+}:\left[\Theta_{0}, \infty\right) \rightarrow\left[\xi_{0}, \infty\right), \nu_{-}:\left[\Theta_{0}, 1\right) \rightarrow\left(-\infty, \xi_{0}\right]$ satisfying

$$
\mu \circ \nu_{ \pm}=\mathrm{id},\left.\quad \nu_{+} \circ \mu\right|_{\left[\xi_{0}, \infty\right)}=\mathrm{id},\left.\quad \nu_{-} \circ \mu\right|_{\left(-\infty, \xi_{0}\right]}=\mathrm{id} .
$$

At some points below it will be technically convenient to extend the functions $\nu_{ \pm}$by $\xi_{0}$ to the interval $\left[0, \Theta_{0}\right)$.

Recalling that the minimum of $\mu$ is non-degenerate one easily establishes 
Lemma 2.1. For any $0<\epsilon \leq 1$ there exists a constant $C>0$ such that for all $0 \leq \beta \leq \beta^{\prime} \leq 1-\epsilon$ one has

$$
0 \leq \nu_{+}\left(\beta^{\prime}\right)-\nu_{+}(\beta) \leq C \sqrt{\beta^{\prime}-\beta}, \quad 0 \leq \nu_{-}(\beta)-\nu_{-}\left(\beta^{\prime}\right) \leq C \sqrt{\beta^{\prime}-\beta} .
$$

As a consequence one obtains the useful estimate

$$
\nu_{+}\left(\beta^{\prime}\right)-\nu_{-}\left(\beta^{\prime}\right) \leq \nu_{+}(\beta)-\nu_{-}(\beta)+2 C \sqrt{\beta^{\prime}-\beta} .
$$

Finally, we denote by $\mu_{1}(\xi)$ the second eigenvalue of the operator (2.1) and put

$$
\Theta_{1}:=\inf _{\xi \in \mathbb{R}} \mu_{1}(\xi) .
$$

Numerically, one finds $\Theta_{1}=2.63 \ldots{ }^{1}$ Below we shall only use the following bound on $\Theta_{1}$, the proof of which is due to B. Helffer.

Lemma 2.2. One has the inequality $\Theta_{1}>1$.

Proof. Denote by $\lambda(\xi)$ the first eigenvalue of the operator (2.1) with a Dirichlet boundary condition at the origin. Then by Sturm-Liouville theory $\mu_{1}(\xi)>\lambda(\xi)$ for all $\xi \in \mathbb{R}$. Moreover, the variational principle implies that

$$
\lambda(\xi) \geq \inf \sigma\left(-\frac{d^{2}}{d t^{2}}+(\xi+t)^{2}\right),
$$

where the operator on the RHS is defined in $L_{2}(\mathbb{R})$. Using translation invariance and the well-known result for the harmonic oscillator one finds that the RHS of (2.3) equals 1.

2.2. Boundary coordinates. Here we would like to recall the definition of coordinates near the boundary of $\Omega$. Recall that we assume that $\partial \Omega$ is connected, $C^{4}$-smooth and of length

$$
\mathcal{L}:=|\partial \Omega| \text {. }
$$

Let $\gamma: \mathbb{R} / \mathcal{L} \mathbb{Z} \rightarrow \partial \Omega$ be a parametrization of the boundary with $\left|\gamma^{\prime}\right| \equiv 1$ and let $\nu(s)$ be the interior unit normal at the point $\gamma(s)$. The parametrization can be chosen such that $\operatorname{det}\left(\gamma^{\prime}, \nu\right) \equiv 1$, so that the curvature $\kappa$ is given by $\kappa(s)=\left\langle\gamma^{\prime \prime}(s), \nu(s)\right\rangle$.

It is well-known that for sufficiently small $t_{0}>0$ the map $\Phi: \mathbb{R} / \mathcal{L} \mathbb{Z} \times$ $\left(0, t_{0}\right) \rightarrow \Omega$,

$$
\Phi(s, t):=\gamma(s)+t \nu(s)
$$

defines a diffeomorphism between $\mathbb{R} / \mathcal{L} \mathbb{Z} \times\left(0, t_{0}\right)$ and its image

$$
\Phi\left(\mathbb{R} / \mathcal{L} \mathbb{Z} \times\left(0, t_{0}\right)\right)=\left\{x \in \Omega: \operatorname{dist}(x, \Omega)<t_{0}\right\}=: \Omega_{t_{0}} .
$$

We now fix a constant $\tilde{\kappa} \in \mathbb{R}$. (For the proof of Theorem 1.1 it will suffice to take $\tilde{\kappa}=0$.) Denoting

$$
a(s, t):=1-t \kappa(s), \quad(s, t) \in \mathbb{R} / \mathcal{L} \mathbb{Z} \times\left(0, t_{0}\right),
$$

\footnotetext{
${ }^{1}$ The author would like to thank V. Bonnaillie-Noël for this calculation.
} 
and

$$
a_{\tilde{\kappa}}(t):=1-t \tilde{\kappa}, \quad t \in \mathbb{R}_{+},
$$

we define for any $u \in L_{2}\left(\Omega_{t_{0}}\right)$

$$
v(s, t):=\left(a(s, t) / a_{\tilde{\kappa}}(t)\right)^{1 / 2} u(\Phi(s, t)), \quad(s, t) \in \mathbb{R} / \mathcal{L} \mathbb{Z} \times\left(0, t_{0}\right) .
$$

This induces a unitary operator from $L_{2}\left(\Omega_{t_{0}}\right)$ to $L_{2}\left((0, \mathcal{L}) \times\left(0, t_{0}\right), a_{\tilde{\kappa}} d s d t\right)$. As in the case $\tilde{\kappa}=0$ considered in HeMo (Appendix B) one finds that if $u \in \mathcal{D}\left[q_{h}\right]$ and $\operatorname{supp} u \subset \overline{\Omega_{t_{0}}}$ then

$$
\begin{aligned}
q_{h}[u]= & \int_{0}^{\mathcal{L}} \int_{0}^{t_{0}}\left(a^{-2}\left|\left(h D_{s}-\tilde{A}_{1}\right) v\right|^{2}+\left|\left(h D_{t}-\tilde{A}_{2}\right) v\right|^{2}+h^{2} W_{\tilde{\kappa}}|v|^{2}\right) a_{\tilde{\kappa}} d s d t \\
& -\frac{h^{2}}{2} \int_{0}^{\mathcal{L}}(\kappa-\tilde{\kappa})|v(., 0)|^{2} d s
\end{aligned}
$$

where

$$
W_{\tilde{\kappa}}(s, t):=-\frac{(\kappa(s)-\tilde{\kappa})(\kappa(s)+\tilde{\kappa}(1-2 t \kappa(s)))}{4 a_{\tilde{\kappa}}(t)^{2} a(s, t)^{2}}-\frac{t \kappa^{\prime \prime}(s)}{2 a(s, t)^{3}}-\frac{5 t^{2} \kappa^{\prime}(s)^{2}}{4 a(s, t)^{4}} .
$$

We do not give the expression for $\tilde{\mathbf{A}}=\left(\tilde{A}_{1}, \tilde{A}_{2}\right)^{T}$ but note only that

$$
\tilde{B}(s, t):=\partial_{s} \tilde{A}_{2}(s, t)-\partial_{t} \tilde{A}_{1}(s, t)=a(s, t) B(\Phi(s, t)) .
$$

\section{Proof of Theorem 1.1}

3.1. The model operator on a half-cylinder. We fix $B, S>0$ and consider the operator $\tilde{P}_{h}^{S, B}=\left(h \mathbf{D}-B \mathbf{A}_{0}\right)^{2}$ in $L_{2}\left((0, S) \times \mathbb{R}_{+}\right)$with periodic boundary conditions at $s \in\{0, S\}$ and Neumann boundary conditions at $t=0$. Here and in the sequel

$$
\mathbf{A}_{0}(s, t):=(-t, 0)^{T} .
$$

Remark 3.1. Since we impose periodic boundary conditions, we actually work on the half-cylinder $\mathbb{R} / S \mathbb{Z} \times \mathbb{R}_{+}$. Since this is a not a simply connected manifold, the magnetic field alone does not determine the operator (up to unitary equivalence), but one also needs to specify the circulation of $\mathbf{A}$ around the boundary $\mathbb{R} / S \mathbb{Z} \times\{0\}$.

We shall use the following notation for a self-adjoint and lower semibounded operator $T$. If $E_{T}(\Lambda), \Lambda \subset \mathbb{R}$, is the spectral measure associated with $T$, we put

$$
N(\lambda, T):=\operatorname{dim} \operatorname{ran} E_{T}((-\infty, \lambda)), \quad \lambda \in \mathbb{R} .
$$

If the spectrum of $T$ below $\lambda$ is discrete, then $N(\lambda, T)$ coincides with the number of eigenvalues (counting multiplicities) below $\lambda$. 
Lemma 3.2. Let $\lambda<h B$. Then

$$
N\left(\lambda, \tilde{P}_{h}^{S, B}\right)=\#\left(\mathbb{Z} \cap \frac{B^{1 / 2} S}{h^{1 / 2} 2 \pi}\left(\nu_{-}\left(h^{-1} B^{-1} \lambda\right), \nu_{+}\left(h^{-1} B^{-1} \lambda\right)\right)\right) .
$$

Recall that the functions $\nu_{ \pm}$are extended by $\xi_{0}$ to $\left[0, \Theta_{0}\right)$. In the statement of the lemma we use the notational convention that $\left(\nu_{-}(\beta), \nu_{+}(\beta)\right)=$ $\left(\xi_{0}, \xi_{0}\right)=\emptyset$ if $\beta \leq \Theta_{0}$.

Proof. By separation of variables the operator $\tilde{P}_{h}^{S, B}$ in $L_{2}\left((0, S) \times \mathbb{R}_{+}\right)$is unitarily equivalent to the direct sum

$$
\sum_{n \in \mathbb{Z}} \oplus\left(-h^{2} \frac{d^{2}}{d t^{2}}+\left(2 \pi n h S^{-1}+B t\right)^{2}\right) \quad \text { in } \sum_{n \in \mathbb{Z}} \oplus L_{2}\left(\mathbb{R}_{+}\right)
$$

(with Neumann boundary conditions at the origin). Applying the dilation $\tau=h^{-1 / 2} B^{1 / 2} t$ we obtain the unitary equivalence

$$
-h^{2} \frac{d^{2}}{d t^{2}}+\left(2 \pi n h S^{-1}+B t\right)^{2} \cong h B\left(-\frac{d^{2}}{d \tau^{2}}+\left(2 \pi n h^{1 / 2} B^{-1 / 2} S^{-1}+\tau\right)^{2}\right) .
$$

By the facts mentioned in Subsection 2.1] (particularly Lemma 2.2) we conclude that for $\lambda<h B$

$$
\begin{aligned}
N\left(\lambda, \tilde{P}_{h}^{S, B}\right) & =\sharp\left\{n \in \mathbb{Z}: \mu\left(2 \pi n h^{1 / 2} B^{-1 / 2} S^{-1}\right)<h^{-1} B^{-1} \lambda\right\} \\
& =\sharp\left(\mathbb{Z} \cap(2 \pi)^{-1} h^{-1 / 2} B^{1 / 2} S\left(\nu_{-}\left(h^{-1} B^{-1} \lambda\right), \nu_{+}\left(h^{-1} B^{-1} \lambda\right)\right)\right),
\end{aligned}
$$

which is what we claimed.

We note that the proof shows that $N\left(h B, \tilde{P}_{h}^{S, B}\right)=+\infty$. Moreover, we easily deduce from Lemma 3.2 that for all $b_{0}<B$

$$
\left|h^{1 / 2} N\left(h b_{0}, \tilde{P}_{h}^{S, B}\right)-\frac{B^{1 / 2} S}{2 \pi}\left(\nu_{+}\left(B^{-1} b_{0}\right)-\nu_{-}\left(B^{-1} b_{0}\right)\right)\right| \leq h^{1 / 2} .
$$

The relation (3.2) is in formal accordance with Theorem 1.1 since

$$
\frac{B^{1 / 2} S}{2 \pi}\left(\nu_{+}\left(B^{-1} b_{0}\right)-\nu_{-}\left(B^{-1} b_{0}\right)\right)=\frac{1}{2 \pi} \iint_{\left\{(s, \xi): B \mu(\xi)<b_{0}\right\}} B^{1 / 2} d s d \xi .
$$

3.2. The model operator on a Dirichlet strip. We fix $S, T, B>0$ and consider the operator $P_{h}^{S, T, B}=\left(h \mathbf{D}-B \mathbf{A}_{0}\right)^{2}$ in $L_{2}((0, S) \times(0, T))$ with Neumann boundary conditions on $t=0$ and Dirichlet boundary conditions on the remaining part of the boundary. Recall that $\mathbf{A}_{0}$ was defined in (3.1). Our goal is to compare the eigenvalue counting function for $P_{h}^{S, T, B}$ with that of $\tilde{P}_{h}^{S, B}$.

Proposition 3.3. For all $B, T, S>0$ and all $\lambda<h B$ one has

$$
N\left(\lambda, P_{h}^{S, T, B}\right) \leq N\left(\lambda, \tilde{P}_{h}^{S, B}\right) .
$$


Moreover, there exists a $C>0$ such that for all $B, T, S>0$, all $\delta \in(0, S / 2]$ and all $\lambda \leq h B$

$$
N\left(\lambda, P_{h}^{S, T, B}\right) \geq \frac{1}{2} N\left(\lambda-C h^{2}\left(\delta^{-2}+T^{-2}\right), \tilde{P}_{h}^{2(S-\delta), B}\right) .
$$

Proof. The extension by zero of a function in the form domain of $P_{h}^{S, T, B}$ lies in the form domain of $\tilde{P}_{h}^{S, B}$, and the values of both forms coincide for such a function. Hence the first assertion follows immediately by the variational principle.

To prove the lower bound we follow the ideas of [CV]. For any $0<\delta \leq S / 2$ we choose a smooth partition of unity on $\mathbb{R} / 2(S-\delta) \mathbb{Z}$,

$$
\left(\varphi_{1}^{\delta}\right)^{2}+\left(\varphi_{2}^{\delta}\right)^{2} \equiv 1 \quad \text { on } \mathbb{R} / 2(S-\delta) \mathbb{Z}
$$

such that

$$
\operatorname{supp} \varphi_{1}^{\delta} \subset[0, S], \quad \operatorname{supp} \varphi_{2}^{\delta} \subset[S-\delta, 2 S-\delta], \quad \sum_{i=1}^{2}\left|\left(\varphi_{i}^{\delta}\right)^{\prime}\right|^{2} \leq c_{1} \delta^{-2} .
$$

The constant $c_{1}>0$ can be chosen independently of $S, \delta$. Similarly, for each $T>0$ let $\psi_{0}, \psi_{1}$ fulfill

$$
\left(\psi_{0}^{T}\right)^{2}+\left(\psi_{1}^{T}\right)^{2} \equiv 1 \quad \text { on } \mathbb{R}_{+}
$$

and

$$
\operatorname{supp} \psi_{0}^{T} \subset[T / 2,+\infty), \quad \operatorname{supp} \psi_{1}^{T} \subset[0, T], \quad \sum_{i=0}^{1}\left|\left(\psi_{i}^{T}\right)^{\prime}\right|^{2} \leq c_{2} T^{-2}
$$

with $c_{2}>0$ independent of $T$. Finally put $\chi_{i}^{\delta, T}(s, t):=\varphi_{i}^{\delta}(s) \psi_{1}^{T}(t), i=1,2$, and $\chi_{0}^{\delta, T}(s, t):=\psi_{0}^{T}(t)$.

Let $u$ be in the form domain of $\tilde{P}_{h}^{2(S-\delta), B}$. Then from the IMS formula with $I^{\delta}:=(0,2(S-\delta))$ we get

$$
\begin{aligned}
\int_{I^{\delta} \times \mathbb{R}_{+}} & \left|\left(h \mathbf{D}-B \mathbf{A}_{0}\right) u\right|^{2} d x \\
= & \sum_{i=0}^{2} \int_{I^{\delta} \times \mathbb{R}_{+}}\left|\left(h \mathbf{D}-B \mathbf{A}_{0}\right) \chi_{i}^{\delta, T} u\right|^{2} d x-h^{2} \sum_{i=0}^{2}\left\|\left|\nabla \chi_{i}^{\delta, T}\right| u\right\|^{2} \\
\geq & \sum_{i=0}^{2} \int_{I^{\delta} \times \mathbb{R}_{+}}\left|\left(h \mathbf{D}-B \mathbf{A}_{0}\right) \chi_{i}^{\delta, T} u\right|^{2} d x-c_{3} h^{2}\left(\delta^{-2}+T^{-2}\right)\|u\|^{2} .
\end{aligned}
$$

The function $\chi_{1}^{\delta, T} u$ belongs to the form domain of $P_{h}^{S, T, B}$ and, since $u$ is periodic, the function $\chi_{2}^{\delta, T} u$ belongs to the form domain of the operator $\tau_{S-\delta} P_{h}^{S, T, B} \tau_{S-\delta}^{*}$ in $L_{2}((S-\delta, 2 S-\delta) \times(0, T))$, where $\tau_{S-\delta}$ denotes translation by $S-\delta$ with respect to the variable $s$. Of course, $\tau_{S-\delta} P_{h}^{S, T, B} \tau_{S-\delta}^{*}$ is unitarily equivalent to $P_{h}^{S, T, B}$. Finally, $\chi_{0}^{\delta, T} u$ belongs to the form domain of the 
operator $\tilde{P}_{h, 0}^{2(S-\delta), B}:=\left(h \mathbf{D}-B \mathbf{A}_{0}\right)^{2}$ in $L_{2}\left(I^{\delta} \times(T / 2, \infty)\right)$ with Dirichlet boundary conditions at $t=T / 2$ and periodic boundary conditions at $s \in$ $\{0,2(S-\delta)\}$. (We do not reflect the dependence on $T$ in our notation, for operators with different $T$ are indeed unitarily equivalent.) Hence we deduce from (3.5) by the variational principle

$$
N\left(\lambda-c_{3} h^{2}\left(\delta^{-2}+T^{-2}\right), \tilde{P}_{h}^{2(S-\delta), B}\right) \leq 2 N\left(\lambda, P_{h}^{S, T, B}\right)+N\left(\lambda, \tilde{P}_{h, 0}^{S, B}\right) .
$$

From an inequality similar to (1.4) with $\Omega$ replaced by $\mathbb{R} / 2(S-\delta) \mathbb{Z} \times \mathbb{R}_{+}$ we infer that $\tilde{P}_{h, 0}^{2(S-\delta), B} \geq h B$. Hence $N\left(\lambda, \tilde{P}_{h, 0}^{S, B}\right)=0$ if $\lambda \leq h B$ and the proposition is proved.

3.3. Estimates near the boundary. Now we consider a general domain $\Omega \subset \mathbb{R}^{2}$ such that $\partial \Omega$ is bounded, connected and $C^{4}$-smooth. We will approximate the quadratic form $q_{h}$ locally near the boundary by a quadratic form corresponding to a constant magnetic field. For this we use the boundary coordinates (as well as the notation) introduced in Subsection 2.2 Throughout this section we will assume that $\tilde{\kappa}=0$.

Let $T \in\left(0, t_{0}\right]$ and $S \in(0, \mathcal{L})$ (below $T, S$ will depend on $h$ and tend to 0 as $h \rightarrow 0)$. We are interested in $u \in \mathcal{D}\left[q_{h}\right]$ such that the corresponding $v$, defined in (2.6), satisfies

$$
\operatorname{supp} v \subset[0, S] \times[0, T] .
$$

First we use a gauge transformation to make the field on $[0, S] \times[0, T]$ 'almost' constant. Indeed, recalling that $\mathbf{A} \in C^{2}\left(\bar{\Omega}, \mathbb{R}^{2}\right)$, one readily obtains

Lemma 3.4. There exists a constant $C>0$ such that for all $S \in(0, \mathcal{L})$, $\tilde{S} \in[0, S]$ there exists a function $\phi \in C^{2}\left([0, S] \times\left[0, t_{0}\right]\right)$ such that

$$
\tilde{\mathbf{A}}(s, t)-\nabla \phi(s, t)=(-\tilde{B} t+\beta(s, t), 0)^{T}, \quad(s, t) \in[0, S] \times\left[0, t_{0}\right],
$$

where $\tilde{B}:=\tilde{B}(\tilde{S}, 0)$ and for any $0<T \leq t_{0}$

$$
\sup _{(s, t) \in[0, S] \times[0, T]}|\beta(s, t)| \leq C\left(S^{2}+T^{2}\right) .
$$

If $u$ is supported in a small subset near the boundary the previous lemma allows us to express $q_{h}[u]$, up to a small error, via a quadratic form corresponding to a constant magnetic field.

Lemma 3.5. There exists a constant $C>0$ such that for all $S \in(0, \mathcal{L})$, $\tilde{S} \in[0, S], T \in\left(0, t_{0}\right], \epsilon \in(0,1]$ satisfying $\epsilon \geq C T$ and for all $u \in \mathcal{D}\left[q_{h}\right]$ such that the corresponding $v$ satisfies (3.6) one has

$$
\begin{aligned}
& \left|q_{h}[u]-\left\|\left(h \mathbf{D}-\tilde{B} \mathbf{A}_{0}\right) e^{-i \phi / h} v\right\|^{2}\right| \\
& \leq \epsilon\left\|\left(h \mathbf{D}-\tilde{B} \mathbf{A}_{0}\right) e^{-i \phi / h} v\right\|^{2}+C \epsilon^{-1}\left(\left(S^{2}+T^{2}\right)^{2}+h^{2}\right)\left\|e^{-i \phi / h} v\right\|^{2} .
\end{aligned}
$$

Here $\tilde{B}:=\tilde{B}(\tilde{S}, 0)$ and $\phi$ is the function from Lemma 3.4 . 
Proof. We write $w:=e^{-i \phi / h} v$. In view of (2.7) we decompose

$$
q_{h}[u]-\left\|\left(h \mathbf{D}-\tilde{B} \mathbf{A}_{0}\right) w\right\|^{2}=I_{1}+I_{2}+I_{3},
$$

where we define

$$
\begin{aligned}
I_{1}:= & \int_{0}^{\mathcal{L}} \int_{0}^{t_{0}}\left(a^{-2}\left|\left(h D_{s}-\tilde{A}_{1}\right) v\right|^{2}+\left|\left(h D_{t}-\tilde{A}_{2}\right) v\right|^{2}\right) d s d t \\
& \quad-\left\|\left(h \mathbf{D}-\tilde{B} \mathbf{A}_{0}\right) w\right\|^{2}, \\
I_{2}:= & h^{2} \int_{0}^{\mathcal{L}} \int_{0}^{t_{0}} W_{0}|v|^{2} d s d t, \\
I_{3}:= & -\frac{h^{2}}{2} \int_{0}^{\mathcal{L}} \kappa|v(., 0)|^{2} d s .
\end{aligned}
$$

We begin with the (easier) terms $I_{2}$ and $I_{3}$. Since $W_{0}$ is bounded, we obtain that for some constant $c_{1}>0$

$$
\left|I_{2}\right| \leq c_{1} h^{2}\|v\|^{2} .
$$

Moreover, for any $s \in[0, L]$ one has

$$
\begin{aligned}
|w(s, 0)|^{2} & =-2 \operatorname{Re} \int_{0}^{T} \frac{\partial}{\partial t} w(s, t) \overline{w(s, t)} d t \\
& \leq \int_{0}^{T}\left(\epsilon_{1}\left|D_{t} w(s, t)\right|^{2}+\epsilon_{1}^{-1}|w(s, t)|^{2}\right) d t .
\end{aligned}
$$

Since $\kappa$ is bounded one easily concludes that there is a constant $c_{2}>0$ such that

$$
\left|I_{3}\right| \leq \epsilon\left\|h D_{t} w\right\|^{2}+c_{2} \epsilon^{-1} h^{2}\|w\|^{2}
$$

for any $\epsilon>0$. Now we turn to the term $I_{1}$. First we note that by Lemma 3.4 one has

$$
I_{1}=\left\|a^{-1}\left(h D_{s}+\tilde{B} t-\beta\right) w\right\|^{2}-\left\|\left(h D_{s}+\tilde{B} t\right) w\right\|^{2} .
$$

We use that for some constant $c_{3}>0$

$$
\left|a^{-2}-1\right| \leq c_{3} T \quad \text { on }[0, S] \times[0, T],
$$

and hence for all $\epsilon_{2}>0$

$$
\begin{aligned}
\left|I_{1}\right| & \leq\left(1+c_{3} T\right)\left\|\left(h D_{s}+\tilde{B} t-\beta\right) w\right\|^{2}-\left\|\left(h D_{s}+\tilde{B} t\right) w\right\|^{2} \\
& \leq\left(\left(1+c_{3} T\right)\left(1+\epsilon_{2}\right)-1\right)\left\|\left(h D_{s}+\tilde{B} t\right) w\right\|^{2}+\left(1+c_{3} T\right)\left(1+\epsilon_{2}^{-1}\right)\|\beta w\|^{2} .
\end{aligned}
$$

In particular, if $\epsilon_{2} \geq T$ then $\left(1+c_{3} T\right)\left(1+\epsilon_{2}\right)-1 \leq c_{4} \epsilon_{2}$. Recalling (3.7) we obtain

$$
\left|I_{1}\right| \leq c_{4} \epsilon_{2}\left\|\left(h D_{s}+\tilde{B} t\right) w\right\|^{2}+c_{5}\left(S^{2}+T^{2}\right)^{2}\left(1+\epsilon_{2}^{-1}\right)\|w\|^{2} .
$$

The assertion now follows easily by setting $c_{4} \epsilon_{2}=\epsilon$ and summing (3.8), (3.9), (3.10). 
3.4. Bracketing. Now we combine Proposition 3.3 and Lemma 3.5 in order to obtain a two-sided estimate of $N\left(h b_{0}, P_{h}\right)$ in terms of the spectral counting functions of operators on a half-cylinder with constant magnetic fields.

For $N \in \mathbb{N}$ put

$$
S:=\frac{\mathcal{L}}{N} \quad \text { and } \quad s_{n}:=n S, n=0, \ldots, N .
$$

We choose

$$
N=\left[h^{-3 / 8}\right],
$$

and note that $S=\mathcal{O}\left(h^{3 / 8}\right)$. Then the core of Theorem 1.1] is contained in

Proposition 3.6. Let $b_{0}<b$. Under the above hypotheses there exists a constant $C>0$ such that for any $0<h \leq C^{-1}, \delta \in(0, S / 2]$ and $\tilde{S}_{n} \in$ $\left[s_{n-1}, s_{n}\right], n=1, \ldots, N$, one has

$$
\begin{aligned}
\frac{1}{2} \sum_{n=1}^{N} N\left(h b_{0}-C h^{2} \delta^{-2}, \tilde{P}_{h}^{2(S-\delta), \tilde{B}_{n}}\right) & \leq N\left(h b_{0}, P_{h}\right) \\
& \leq \sum_{n=1}^{N} N\left(h b_{0}+C h^{2} \delta^{-2}, \tilde{P}_{h}^{S+2 \delta, \tilde{B}_{n}}\right)
\end{aligned}
$$

where $\tilde{B}_{n}:=\tilde{B}\left(\tilde{S}_{n}, 0\right)$.

Proof. We begin with the proof of the lower bound. We apply Lemma 3.5 with

$$
T=h^{3 / 8}, \quad \epsilon=h^{1 / 4}
$$

not only on $[0, S]$, but on any $\left[s_{n-1}, s_{n}\right]$. (It is evident from the proof of that lemma that the constants there can be chosen independently of $n$.) Moreover, recall that $S=\mathcal{O}\left(h^{3 / 8}\right)$. It follows that, for some constant $c_{1}>0$ and for all $u \in \mathcal{D}\left[q_{h}\right]$ which vanish on

$$
\{x \in \Omega: t(x) \geq T\} \cup \bigcup_{n=1}^{N}\left\{x \in \Omega: 0 \leq t(x) \leq T, s(x)=s_{n}\right\},
$$

one has the estimate

$$
\begin{aligned}
q_{h}[u] \leq & \left(1+h^{1 / 4}\right) \sum_{n=1}^{N} \int_{s_{n-1}}^{s_{n}} \int_{0}^{T}\left|\left(h \mathbf{D}-\tilde{B}_{n} \mathbf{A}_{0}\right) e^{-i \phi_{n} / h} v\right|^{2} d s d t \\
& +c_{1} h^{5 / 4}\left\|e^{-i \phi_{n} / h} v\right\|^{2},
\end{aligned}
$$

where $v$ is defined by (2.6). Hence by the variational principle

$$
N\left(\lambda, P_{h}\right) \geq \sum_{n=1}^{N} N\left(\frac{\lambda-c_{1} h^{5 / 4}}{1+h^{1 / 4}}, P_{h}^{S, T, \tilde{B}_{n}}\right) .
$$

We apply Proposition 3.3 with $\delta \in(0, S / 2]$. Since

$$
\frac{h b_{0}-c_{1} h^{5 / 4}}{1+h^{1 / 4}}-C h^{2}\left(\delta^{-2}+T^{-2}\right) \geq h b_{0}-c_{2} h^{2} \delta^{-2},
$$


we obtain

$$
N\left(h b_{0}, P_{h}\right) \geq \frac{1}{2} \sum_{n=1}^{N} N\left(h b_{0}-c_{2} h^{2} \delta^{-2}, \tilde{P}_{h}^{2(S-\delta), \tilde{B}_{n}}\right) .
$$

This is the desired lower bound.

We turn to the proof of the upper bound. We choose for every $\delta \in(0, S / 2]$ a partition of unity on $\mathbb{R} / \mathcal{L} \mathbb{Z}$,

$$
\sum_{i=1}^{N}\left(\varphi_{n}^{\delta}\right)^{2} \equiv 1 \text { on } \mathbb{R} / \mathcal{L} \mathbb{Z} \quad \text { with } \quad \operatorname{supp} \varphi_{n}^{\delta} \subset\left[s_{n-1}-\delta, s_{n}+\delta\right]
$$

and such that

$$
\sum_{i=1}^{N}\left|\left(\varphi_{n}^{\delta}\right)^{\prime}\right|^{2} \leq c_{3} \delta^{-2}
$$

The constant $c_{3}>0$ can be chosen independent of $\delta, S, N$. Moreover, let $\psi_{0}^{T}, \psi_{1}^{T}$ be as in (3.3), (3.4), and for $T \in\left(0, t_{0}\right)$ put

$$
\chi_{n}^{\delta, T}(x):=\varphi_{n}^{\delta}(s(x)) \psi_{1}^{T}(t(x)), n=1, \ldots, N, \quad \chi_{0}^{\delta, T}(x):=\psi_{0}^{T}(t(x)) .
$$

By means of the IMS formula we find for all $u \in \mathcal{D}\left[q_{h}\right]$

$$
\begin{aligned}
q_{h}[u] & =\sum_{n=0}^{N} q_{h}\left[\chi_{n}^{\delta, T} u\right]-h^{2} \sum_{n=0}^{N}\left\|\left|\nabla \chi_{n}^{\delta, T}\right| u\right\|^{2} \\
& \geq \sum_{n=0}^{N} q_{h}\left[\chi_{n}^{\delta, T} u\right]-c_{4} h^{2}\left(\delta^{-2}+T^{-2}\right)\|u\|^{2} .
\end{aligned}
$$

As in the proof of the upper bound choose $T, \epsilon$ as in (3.4). Then we obtain from Lemma 3.5 that for $n=1, \ldots, N$

$$
\begin{aligned}
q_{h}\left[\chi_{n}^{\delta, T} u\right] \geq( & \left.1-h^{1 / 4}\right) \int_{s_{n-1}}^{s_{n}} \int_{0}^{T}\left|\left(h \mathbf{D}-\tilde{B}_{n} \mathbf{A}_{0}\right) e^{-i \phi_{n} / h} \chi_{n}^{\delta, T} v\right|^{2} d s d t \\
& -c_{5} h^{5 / 4}\left\|e^{-i \phi_{n} / h} \chi_{n}^{\delta, T} v\right\|^{2},
\end{aligned}
$$

where $v$ is related to $u$ by (2.6) and where we write $\chi_{n}^{\delta, T}$ on the RHS instead of $\chi_{n}^{\delta, T} \circ \Phi$. It follows that

$$
\begin{aligned}
q_{h}[u] \geq & \left(1-h^{1 / 4}\right) \sum_{n=1}^{N} \int_{s_{n-1}}^{s_{n}} \int_{0}^{T}\left|\left(h \mathbf{D}-\tilde{B}_{n} \mathbf{A}_{0}\right) e^{-i \phi_{n} / h} \chi_{n}^{\delta, T} v\right|^{2} d s d t \\
& +q_{h}\left[\chi_{0}^{\delta, T} u\right]-c_{6} h^{2} \delta^{-2}\|u\|^{2},
\end{aligned}
$$

and hence by the variational principle

$$
N\left(\lambda, P_{h}\right) \leq \sum_{n=1}^{N} N\left(\frac{\lambda+c_{6} h^{2} \delta^{-2}}{1-h^{1 / 4}}, P_{h}^{S+2 \delta, T, \tilde{B}_{n}}\right)+N\left(\lambda+c_{6} h^{2} \delta^{-2}, P_{h, 0}^{T}\right),
$$


where $P_{h, 0}^{T}:=(h \mathbf{D}-\mathbf{A})^{2}$ in $L_{2}\left(\Omega \backslash \overline{\Omega_{T / 2}}\right)$ with Dirichlet boundary conditions. Because of the inequality (1.4) we have $N\left(\lambda, P_{h, 0}^{T}\right)=0$ for $\lambda \leq h b$. Moreover, since

$$
\frac{h b_{0}+c_{6} h^{2} \delta^{-2}}{1-h^{1 / 4}} \leq h b_{0}+c_{7} h^{2} \delta^{-2},
$$

the desired upper bound follows from Proposition 3.3 .

3.5. Proof of Theorem 1.1. To complete the proof of our main result we now combine Proposition 3.6 with the explicit calculation in Lemma 3.2 for the half-cylinder. Indeed, instead of (1.7) we prove the stronger (albeit probably not sharp) estimate

$$
h^{1 / 2} N\left(h b_{0}, P_{h}\right)=\frac{1}{2 \pi} \int_{\partial \Omega} \int_{\left\{\xi: \mu(\xi)<\frac{b_{0}}{B(x)}\right\}} B(x)^{1 / 2} d \xi d s(x)+\mathcal{O}\left(h^{1 / 16}\right)
$$

for $b_{0}<b$. The proofs of the upper and the lower bound in (3.12) are similar and we only give the latter one.

Let $N, S$ be as in Subsection 3.4 and let $\tilde{S}_{n} \in\left[s_{n-1}, s_{n}\right]$ be arbitrary with $\tilde{B}_{n}:=\tilde{B}\left(\tilde{S}_{n}, 0\right)$. Note that for all sufficiently small $h$ one has $b_{0}-C h \delta^{-2}<$ $b \leq b^{\prime} \leq \tilde{B}_{n}$. Hence, from (3.2) and Proposition 3.6, for all $\delta \in(0, S / 2]$

$$
\begin{aligned}
h^{1 / 2} N\left(h b_{0}, P_{h}\right) \geq \frac{1}{2 \pi} & \sum_{n=1}^{N}(S-\delta) \tilde{B}_{n}^{1 / 2} \times \\
& \times\left(\nu_{+}\left(\tilde{B}_{n}^{-1}\left(b_{0}-C h \delta^{-2}\right)\right)-\nu_{-}\left(\tilde{B}_{n}^{-1}\left(b_{0}-C h \delta^{-2}\right)\right)\right) \\
& -\frac{N}{2} h^{1 / 2} .
\end{aligned}
$$

Now we use (2.2), choose $\delta=h^{7 / 16}$ and recall that $N=\mathcal{O}\left(h^{-3 / 8}\right)$ to get

$$
h^{1 / 2} N\left(h b_{0}, P_{h}\right) \geq \frac{1}{2 \pi} \sum_{n=1}^{N} S \tilde{B}_{n}^{1 / 2}\left(\nu_{+}\left(\tilde{B}_{n}^{-1} b_{0}\right)-\nu_{-}\left(\tilde{B}_{n}^{-1} b_{0}\right)\right)-c_{1} h^{1 / 16} .
$$

The main term on the RHS is a Riemannian sum. Recalling that the $\tilde{S}_{n}$ were arbitrary, we finally arrive at

$$
\begin{array}{rl}
h^{1 / 2} & N\left(h b_{0}, P_{h}\right) \\
& \geq \frac{1}{2 \pi} \int_{\partial \Omega} B(x)^{1 / 2}\left(\nu_{+}\left(\frac{b_{0}}{B(x)}\right)-\nu_{-}\left(\frac{b_{0}}{B(x)}\right)\right) d s(x)-c_{1} h^{1 / 16} \\
& =\frac{1}{2 \pi} \int_{\partial \Omega} \int_{\left\{\xi: \mu(\xi)<\frac{b_{0}}{B(x)}\right\}} B(x)^{1 / 2} d \xi d s(x)-c_{1} h^{1 / 16} .
\end{array}
$$

This is the lower bound of (3.12). In a similar fashion one can establish the upper bound, which concludes the proof of Theorem 1.1. 


\section{Proof of Theorem 1.2}

4.1. The model operator on a half-cylinder. For parameters $S, T>0$, $\kappa \in \mathbb{R}$ satisfying

$$
2|\kappa| T \leq 1,
$$

we denote by $\tilde{M}_{h}^{S, T, \kappa}$ the self-adjoint operator in $L_{2}\left((0, S) \times(0, T), a_{\kappa} d s d t\right)$ associated with the quadratic form

$$
\begin{aligned}
\tilde{m}_{h}^{S, T, \kappa}[u] & :=\int_{0}^{S} \int_{0}^{T}\left(a_{\kappa}^{-2}\left|\left(h D_{s}+t-\kappa t^{2} / 2\right) u\right|^{2}+\left|h D_{t} u\right|^{2}\right) a_{\kappa} d s d t, \\
\mathcal{D}\left[\tilde{m}_{h}^{S, T, \kappa}\right]: & :=\left\{u \in H^{1}((0, S) \times(0, T)): u(., T)=0, u(0, .)=u(S, .)\right\} .
\end{aligned}
$$

Recall that the function $a_{\kappa}$ was defined in (2.5). (We emphasize once more that here and in the next subsection, $\kappa$ will be a constant and not the curvature.)

Moreover, recall the constant $\mathcal{C}_{1}$ from (1.5). The goal of this subsection is to prove

Proposition 4.1. Let $D>0$. Then there exist $C, \epsilon>0$ such that for all $|\kappa| \leq D,\left|\kappa_{0}\right| \leq D, S>0,0<h \leq \epsilon$ and $\epsilon^{-1} \sqrt{h}|\log h| \leq T \leq \epsilon h^{1 / 4}$ one has

$$
\left|N\left(h \Theta_{0}+h^{3 / 2} \mathcal{C}_{1} \kappa_{0}, \tilde{M}_{h}^{S, T, \kappa}\right)-h^{-1 / 4} \frac{S}{\pi \sqrt{3\left|\xi_{0}\right|}}\left(\kappa+\kappa_{0}\right)_{+}^{1 / 2}\right| \leq C .
$$

For the analysis of the operators $\tilde{M}_{h}^{S, T, \kappa}$ we begin as in the proof of Lemma 3.2. By separation of variables and a dilation $\tau=h^{-1 / 2} t$ we obtain the unitary equivalence

$$
\tilde{M}_{h}^{S, T, \kappa} \cong h \sum_{n \in \mathbb{Z}} \oplus M\left(2 \pi n h^{1 / 2} S^{-1}, h^{1 / 2} \kappa, h^{-1 / 2} T\right)
$$

Here we define, for parameters

$$
\xi \in \mathbb{R}, \quad \alpha \in[-1,1], \quad L \geq 1, \quad 2|\alpha| L \leq 1,
$$

the self-adjoint operator $M(\xi, \alpha, L)$ in the Hilbert space $L_{2}\left((0, L), a_{\kappa} d \tau\right)$ by the quadratic form

$$
\begin{aligned}
& m(\xi, \alpha, L)[f]:=\int_{0}^{L}\left(\left|f^{\prime}\right|^{2}+a_{\alpha}^{-2}\left(\xi+\tau-\alpha \tau^{2} / 2\right)^{2}|f|^{2}\right) a_{\alpha} d \tau, \\
& \mathcal{D}[m(\xi, \alpha, L)]:=\left\{f \in H^{1}(0, L): f(L)=0\right\} .
\end{aligned}
$$

The proof of Proposition 4.1 relies on the following two results, which we take from HeMo (Section 11), see also [FoHe1] (Lemma 5.4). We shall denote the eigenvalues of a self-adjoint and lower semibounded operator $T$ with compact resolvent by $\mu_{1}(T) \leq \mu_{2}(T) \leq \ldots$, taking multiplicities into account. 
Lemma 4.2. Let $D>0$. Then there exists a $C>0$ such that if $|\alpha| L^{2} \leq D$ then for all $j \in \mathbb{N}$

$$
\left|\mu_{j}(M(\xi, \alpha, L))-\mu_{j}(M(\xi, 0, L))\right| \leq C|\alpha| L^{2}\left(1+\mu_{j}(M(\xi, 0, L))\right) .
$$

The previous lemma follows easily by comparing the corresponding quadratic forms. Using an explicit trial function one can show

Lemma 4.3. Let $D>0$. Then there exists a $C>0$ such that for all $\left|\xi-\xi_{0}\right| \leq D, L \geq C$ there exists a $\lambda \in \sigma(M(\xi, \alpha, L))$ with

$$
\begin{aligned}
\mid \lambda- & \Theta_{0}-3 \mathcal{C}_{1}\left|\xi_{0}\right|\left(\xi-\xi_{0}\right)^{2}+\mathcal{C}_{1} \alpha \mid \\
& \leq C\left(\left|\xi-\xi_{0}\right|^{3}+|\alpha|\left|\xi-\xi_{0}\right|+\alpha^{2}+e^{-L / C}\right) .
\end{aligned}
$$

We will use the following consequence of the two preceding lemmas.

Corollary 4.4. Let $\beta>0$. Then there exist $\epsilon, \delta, C>0$ such that for all $\xi \in \mathbb{R},|\alpha| L^{2} \leq \epsilon, L \geq \epsilon^{-1}$ one has:

(1) If $\left|\xi-\xi_{0}\right| \leq \beta C^{-1}$ then

$$
\begin{array}{r}
\left|\mu_{1}(M(\xi, \alpha, L))-\Theta_{0}-3 \mathcal{C}_{1}\right| \xi_{0}\left|\left(\xi-\xi_{0}\right)^{2}+\mathcal{C}_{1} \alpha\right| \\
\leq C\left(\left|\xi-\xi_{0}\right|^{3}+|\alpha|\left|\xi-\xi_{0}\right|+\alpha^{2}+e^{-L / C}\right)
\end{array}
$$

and

(2) If $\left|\xi-\xi_{0}\right| \geq \beta C^{-1}$ then

$$
\mu_{2}(M(\xi, \alpha, L)) \geq \Theta_{0}+\delta
$$

$$
\mu_{1}(M(\xi, \alpha, L)) \geq \Theta_{0}+\delta
$$

The parameter $\beta$ is introduced for technical reasons which will become clear in the proof of Proposition 4.1.

Proof of Corollary 4.4. By Lemma 4.3 there exist $\epsilon, C>0$ such that if $\mid \xi-$ $\xi_{0}\left|\leq \beta C^{-1},\right| \alpha \mid L^{2} \leq \epsilon$ and $L \geq \epsilon^{-1}$ then $M(\xi, \alpha, L)$ has an eigenvalue $\lambda$ below $\frac{1}{2}\left(\Theta_{0}+\Theta_{1}\right)$ satisfying (4.2) with the constant $C$. (We could of course choose $\epsilon=C^{-1}$ or $\epsilon=\beta C^{-1}$, but later it will be useful to keep them separated.) Note that by the variational principle we have

$$
\mu_{j}(M(\xi, 0, L)) \geq \mu_{j}(M(\xi))
$$

where $M(\xi)$ denotes the operator studied in Subsection 2.1 (corresponding to $\alpha=0$ and $L=\infty$ ). Hence by Lemma 4.2 we find that, after decreasing $\epsilon$ if necessary, one has for all $\left|\xi-\xi_{0}\right| \leq \beta C^{-1}$

$$
\begin{aligned}
\mu_{2}(M(\xi, \alpha, L)) & \geq\left(1-c_{1}|\alpha| L^{2}\right) \mu_{2}(M(\xi, 0, L))-c_{1}|\alpha| L^{2} \\
& \geq\left(1-c_{1}|\alpha| L^{2}\right) \mu_{2}(M(\xi))-c_{1}|\alpha| L^{2} \\
& \geq\left(1-c_{1}|\alpha| L^{2}\right) \Theta_{1}-c_{1}|\alpha| L^{2} \\
& \geq\left(\Theta_{0}+\Theta_{1}\right) / 2 .
\end{aligned}
$$

In particular, it follows that $\lambda=\mu_{1}(M(\xi, \alpha, L))$. This finishes the proof of the first part of the corollary (with $\delta \leq \frac{1}{2}\left(\Theta_{1}-\Theta_{0}\right)$ arbitrary). 
By the properties of the function $\mu$ recalled in Subsection 2.1 it is clear that there exists a constant $\delta>0$ such that for all $\left|\xi-\xi_{0}\right| \geq \beta C^{-1}$ one has

$$
\mu(\xi) \geq \Theta_{0}+2 \delta .
$$

(We can assume that $\delta \leq \frac{1}{2}\left(\Theta_{0}+\Theta_{1}\right)$.) Applying again (4.3) and Lemma4.2 and decreasing $\epsilon$ if necessary we find for all $\left|\xi-\xi_{0}\right| \geq \beta C^{-1}$

$$
\begin{aligned}
\mu_{1}(M(\xi, \alpha, L)) & \geq\left(1-c_{1}|\alpha| L^{2}\right) \mu_{1}(M(\xi, 0, L))-c_{1}|\alpha| L^{2} \\
& \geq\left(1-c_{1}|\alpha| L^{2}\right) \mu(\xi)-c_{1}|\alpha| L^{2} \\
& \geq\left(1-c_{1}|\alpha| L^{2}\right)\left(\Theta_{0}+2 \delta\right)-c_{1}|\alpha| L^{2} \\
& \geq \Theta_{0}+\delta .
\end{aligned}
$$

This finishes the proof of the second part of the corollary.

Everything is now in place for the

Proof of Proposition 4.1. We keep the notation $\epsilon, \delta, C$ for the constants from Corollary 4.4 corresponding to

$$
\beta:=3 \mathcal{C}_{1}\left|\xi_{0}\right| / 2
$$

and we will assume that

$$
C \sqrt{h}|\log h| \leq T \leq \sqrt{\epsilon / D} h^{1 / 4} .
$$

Then there exists a $h_{0}>0$ such that for all $0<h \leq h_{0}$ and all $|\kappa|,\left|\kappa_{0}\right| \leq$ $D$ one has $h^{-1 / 2}|\kappa| T^{2} \leq \epsilon, h^{-1 / 2} T \geq \epsilon^{-1}$ and $h^{1 / 2} \mathcal{C}_{1} \kappa_{0} \leq \delta$. Therefore Corollary 4.4 and the decomposition (4.1) imply that

$$
\begin{aligned}
& N\left(h \Theta_{0}+h^{3 / 2} \mathcal{C}_{1} \kappa_{0}, \tilde{M}_{h}^{S, T, \kappa}\right) \\
& \quad=\sharp\left\{n \in \mathbb{Z}: \mu_{1}\left(M\left(2 \pi n h^{1 / 2} S^{-1}, h^{1 / 2} \kappa, h^{-1 / 2} T\right)\right)<\Theta_{0}+h^{1 / 2} \mathcal{C}_{1} \kappa_{0}\right\} .
\end{aligned}
$$

Noting that $h \kappa^{2}+e^{-T / C \sqrt{h}} \leq h\left(D^{2}+1\right)$ we find from Corollary 4.4 the estimates

$$
\begin{gathered}
\sharp\left\{n \in \mathbb{Z}: p_{+}\left(\left|2 \pi n S^{-1}-h^{-1 / 2} \xi_{0}\right|\right)<0\right\} \\
\leq N\left(h \Theta_{0}+h^{3 / 2} \mathcal{C}_{1} \kappa_{0}, \tilde{M}_{h}^{S, T, \kappa}\right) \\
\leq \sharp\left\{n \in \mathbb{Z}:\left|2 \pi n S^{-1}-h^{-1 / 2} \xi_{0}\right| \leq \beta C^{-1} h^{-1 / 2},\right. \\
\left.p_{-}\left(\left|2 \pi n S^{-1}-h^{-1 / 2} \xi_{0}\right|\right)<0\right\}
\end{gathered}
$$

where

$$
p_{ \pm, h}(y):=3 \mathcal{C}_{1}\left|\xi_{0}\right| y^{2}-\mathcal{C}_{1} h^{-1 / 2}\left(\kappa+\kappa_{0}\right) \pm C\left(h^{1 / 2} y^{3}+D y+D^{2}+1\right) .
$$

The assertion will follow from the properties of these polynomials which we will discuss now briefly.

We start with $p_{+, h}$. If $\kappa+\kappa_{0} \leq \mathcal{C}_{1}^{-1} C\left(D^{2}+1\right) h^{1 / 2}$ one has $p_{+, h}(y)>0$ for all $y>0$ and we define $y_{+, h}:=0$. On the other hand, if $\kappa+\kappa_{0}>$ 
$\mathcal{C}_{1}^{-1} C\left(D^{2}+1\right) h^{1 / 2}$, one checks that there is a unique zero $y_{+, h} \in(0, \infty)$, and that this satisfies

$$
y_{+, h}=\left(3\left|\xi_{0}\right|\right)^{-1 / 2}\left(\kappa+\kappa_{0}\right)_{+}^{1 / 2} h^{-1 / 4}+\mathcal{O}(1)
$$

as $h \rightarrow 0$, where $\mathcal{O}(1)$ is uniform in $\kappa, \kappa_{0}$ (varying in a bounded set). Hence in any case, we obtain from (4.5) the lower bound

$$
\begin{aligned}
N\left(h \Theta_{0}+h^{3 / 2} \mathcal{C}_{1} \kappa_{0}, \tilde{M}_{h}^{S, T, \kappa}\right) & \geq \sharp\left\{n \in \mathbb{Z}:\left|2 \pi n S^{-1}-h^{-1 / 2} \xi_{0}\right|<y_{+, h}\right\} \\
& =h^{-1 / 4} \frac{S}{\pi \sqrt{3\left|\xi_{0}\right|}}\left(\kappa+\kappa_{0}\right)_{+}^{1 / 2}+\mathcal{O}(1) .
\end{aligned}
$$

Now we turn to the polynomial $p_{-, h}$. First we note that there is a zero $\tilde{y}_{-, h} \sim 3 \mathcal{C}_{1}\left|\xi_{0}\right| C^{-1} h^{-1 / 2}$, which however does not lie in the interval $\left[0, \beta C^{-1} h^{-1 / 2}\right)$ if $h$ is sufficiently small. (This is the reason for the choice of $\beta$ in (4.4) $)$.) If $\kappa+\kappa_{0} \leq-\mathcal{C}_{1}^{-1} C\left(D^{2}+1\right) h^{1 / 2}, \tilde{y}_{-, h}$ is the only zero in $(0, \infty)$ and we set $y_{-, h}:=0$. On the other hand, if $\kappa+\kappa_{0}>-\mathcal{C}_{1}^{-1} C\left(D^{2}+1\right) h^{1 / 2}$, one checks that there is a unique zero $y_{-, h} \in\left[0, \tilde{y}_{-, h}\right)$, and that this has the same expansion as in (4.6). In both cases, we obtain the upper bound

$$
\begin{aligned}
N\left(h \Theta_{0}+h^{3 / 2} \mathcal{C}_{1} \kappa_{0}, \tilde{M}_{h}^{S, T, \kappa}\right) & \leq \sharp\left\{n \in \mathbb{Z}:\left|2 \pi n S^{-1}-h^{-1 / 2} \xi_{0}\right|<y_{-, h}\right\} \\
& =h^{-1 / 4} \frac{S}{\pi \sqrt{3\left|\xi_{0}\right|}}\left(\kappa+\kappa_{0}\right)_{+}^{1 / 2}+\mathcal{O}(1) .
\end{aligned}
$$

This proves the assertion.

4.2. The model operator on a Dirichlet strip. We fix $S, T, \kappa$ as in the previous subsection and consider the operator $M_{h}^{S, T, \kappa}$ obtained from $\tilde{M}_{h}^{S, T, \kappa}$ by imposing additional Dirichlet boundary conditions at $s \in\{0, S\}$. More precisely, $M_{h}^{S, T, \kappa}$ is the self-adjoint operator in $L_{2}\left((0, S) \times(0, T), a_{\kappa} d s d t\right)$ associated with the quadratic form

$$
\begin{aligned}
m_{h}^{S, T, \kappa}[u] & :=\int_{0}^{S} \int_{0}^{T}\left(a_{\kappa}^{-2}\left|\left(h D_{s}+t-\kappa t^{2} / 2\right) u\right|^{2}+\left|h D_{t} u\right|^{2}\right) a_{\kappa} d s d t, \\
\mathcal{D}\left[m_{h}^{S, T, \kappa}\right] & :=\left\{u \in H^{1}((0, S) \times(0, T)): u(., T)=u(0, .)=u(S, .)=0\right\} .
\end{aligned}
$$

With an argument similar to that in Subsection 3.2 one proves

Proposition 4.5. There exists a $C>0$ such that for all $S, T>0, \kappa \in \mathbb{R}$ with $2|\kappa| T \leq 1$ and all $\lambda \in \mathbb{R}$ and $\delta \in(0, S / 2]$ one has

$$
\frac{1}{2} N\left(\lambda-C h^{2} \delta^{-2}, \tilde{M}_{h}^{2(S-\delta), T, \kappa}\right) \leq N\left(\lambda, M_{h}^{S, T, \kappa}\right) \leq N\left(\lambda, \tilde{M}_{h}^{S, T, \kappa}\right) .
$$

4.3. Estimates near the boundary. Similarly as in Subsection 3.3 we will now approximate the quadratic form $q_{h}$ locally near the boundary but, the magnetic field now being constant, this can be done with a higher precision. In particular we will see the curvature of the boundary appear. Again we shall use the notation from Subsection 2.2 and, in contrast to Subsection 3.3. it will be important now to keep $\tilde{\kappa}$ arbitrary. 
We will assume in this subsection that $B \equiv 1$. Then we can choose the magnetic vector potential in the following way.

Lemma 4.6. There exists a constant $C>0$ such that for all $S \in(0, L)$, $\tilde{S} \in[0, S]$ there exists a $\phi \in C^{2}\left([0, S] \times\left[0, t_{0}\right]\right)$ such that

$$
\tilde{\mathbf{A}}(s, t)-\nabla \phi(s, t)=\left(-t+\tilde{\kappa} t^{2} / 2+\beta(s, t), 0\right)^{T}, \quad(s, t) \in[0, S] \times\left[0, t_{0}\right],
$$

where $\tilde{\kappa}:=\kappa(\tilde{S})$ and for any $0<T \leq t_{0}$

$$
\sup _{(s, t) \in[0, S] \times[0, T]}|\beta(s, t)| \leq C S T^{2} .
$$

Indeed, one can take $\phi(s, t):=\int_{0}^{t} \partial_{s} \tilde{A}_{2}\left(s, t^{\prime}\right) d t^{\prime}-\int_{0}^{s} \tilde{A}_{1}\left(s^{\prime}, 0\right) d s^{\prime}$ and recall that $\partial_{s} \tilde{A}_{2}(s, t)-\partial_{t} \tilde{A}_{1}(s, t)=1-t \kappa(s)$.

Before stating the next result we recall the definition of $v$ from (2.6) and of the quadratic form $m_{h}^{S, T, \kappa}$ from Subsection 4.2

Lemma 4.7. Let $D>0$. Then there exists a constant $C>0$ such that for all $S \in(0, L), \tilde{S} \in[0, S], T \in\left(0, t_{0}\right]$ with $T \geq D \sqrt{h}$ and for all $u \in \mathcal{D}\left[q_{h}\right]$ such that the corresponding $v$ satisfies (3.6) one has

$$
\begin{aligned}
\mid q_{h}[u] & -m_{h}^{S, T, \tilde{\kappa}}\left[e^{-i \phi / h} v\right] \mid \\
& \leq C\left(S T m_{h}^{S, T, \tilde{\kappa}}\left[e^{-i \phi / h} v\right]+\left(h^{2} T+S T^{3}\right)\left\|e^{-i \phi / h} v\right\|_{\tilde{\kappa}}^{2}\right) .
\end{aligned}
$$

Here $\tilde{\kappa}:=\kappa(\tilde{S})$ and $\phi$ is the function from Lemma 4.6. Moreover, $\|\cdot\|_{\tilde{\kappa}}$ denotes the norm in $L_{2}\left((0, S) \times(0, T), a_{\tilde{\kappa}} d s d t\right)$.

Proof. The proof is rather similar to that of Lemma 3.5 so we will only sketch the major steps. Writing $w:=e^{-i \phi / h} v$ and taking (2.7) into account we decompose

$$
q_{h}[u]-m_{h}^{S, T, \tilde{\kappa}}[w]=I_{1}+I_{2}+I_{3}
$$

where we define

$$
\begin{aligned}
I_{1}:= & \int_{0}^{\mathcal{L}} \int_{0}^{t_{0}}\left(a^{-2}\left|\left(h D_{s}-\tilde{A}_{1}\right) v\right|^{2}+\left|\left(h D_{t}-\tilde{A}_{2}\right) v\right|^{2}\right) a_{\tilde{\kappa}} d s d t \\
& \quad-m_{h}^{S, T, \tilde{\kappa}}[w], \\
I_{2}:= & h^{2} \int_{0}^{\mathcal{L}} \int_{0}^{t_{0}} W_{\tilde{\kappa}}|v|^{2} a_{\tilde{\kappa}} d s d t, \\
I_{3}:= & -\frac{h^{2}}{2} \int_{0}^{\mathcal{L}}(\kappa-\tilde{\kappa})|v(., 0)|^{2} d s .
\end{aligned}
$$

To treat the terms $I_{2}$ and $I_{3}$ we use that $|\kappa-\tilde{\kappa}| \leq c_{1} S$ on the support of $w$. This leads to the estimates

$$
\begin{aligned}
& \left|I_{2}\right| \leq c_{2} h^{2}(S+T)\|w\|_{\tilde{\kappa}}^{2}, \\
& \left|I_{3}\right| \leq \epsilon\left\|h D_{t} w\right\|_{\tilde{\kappa}}^{2}+c_{3} \epsilon^{-1} h^{2} S^{2}\|w\|_{\tilde{\kappa}}^{2} .
\end{aligned}
$$


for any $\epsilon>0$. To take care of $I_{1}$ we note that by Lemma 4.6 one has

$$
\begin{aligned}
I_{1}=\int_{0}^{\mathcal{L}} \int_{0}^{t_{0}}\left(a^{-2}\left|\left(h D_{s}+t-\tilde{\kappa} t^{2} / 2-\beta\right) w\right|^{2}\right. \\
\\
\left.\quad-a_{\tilde{\kappa}}^{-2}\left|\left(h D_{s}+t-\tilde{\kappa} t^{2} / 2\right) w\right|^{2}\right) a_{\tilde{\kappa}} d s d t .
\end{aligned}
$$

Using the estimate on $\beta$ from (4.7) and that $\left|a^{-2} a_{\tilde{\kappa}}^{2}-1\right| \leq c_{4} S T$ on the support of $w$ we easily find, for all $\epsilon>0$,

$$
\begin{aligned}
\left|I_{1}\right| \leq( & \left.+c_{5} S T\right) \int_{0}^{\mathcal{L}} \int_{0}^{t_{0}} a_{\tilde{\kappa}}^{-2}\left|\left(h D_{s}+t-\tilde{\kappa} t^{2} / 2\right) w\right|^{2} a_{\tilde{\kappa}} d s d t \\
& +c_{5}\left(1+\epsilon^{-1}\right) S^{2} T^{4}\|w\|_{\tilde{\kappa}}^{2} .
\end{aligned}
$$

The assertion then follows by choosing $\epsilon=S T$ and recalling $T \geq D \sqrt{h}$.

4.4. Bracketing. Now we estimate $N\left(h \Theta_{0}+h^{3 / 2} \mathcal{C}_{1} \kappa_{0}, P_{h}\right)$ by the spectral counting functions of the operators with constant curvature from Subsection 4.1. Again we assume that $B \equiv 1$.

For $N \in \mathbb{N}$ we define $S$ and $s_{n}$ as in (3.11). In contrast to Subsection 3.4 we will not yet specify the value of $N$ but postpone this to the next subsection.

Proposition 4.8. Let $\kappa_{0} \in \mathbb{R}$. Under the above hypotheses there exists a constant $C>0$ such that for any $0<h \leq C^{-1}, \delta \in(0, S / 2]$, T with $C h^{1 / 2}|\log h| \leq T \leq C^{-1} h^{1 / 4}$ and $\tilde{S}_{n} \in\left[s_{n-1}, s_{n}\right], n=1, \ldots, N$, one has

$$
\begin{array}{rl}
\frac{1}{2} \sum_{n=1}^{N} & N\left(h \Theta_{0}+h^{3 / 2} \mathcal{C}_{1} \kappa_{0}-C\left(S T^{3}+h^{2} \delta^{-2}\right), \tilde{M}_{h}^{2(S+\delta), T, \tilde{\kappa}_{n}}\right) \\
& \leq N\left(h \Theta_{0}+h^{3 / 2} \mathcal{C}_{1} \kappa_{0}, P_{h}\right) \\
& \leq \sum_{n=1}^{N} N\left(h \Theta_{0}+h^{3 / 2} \mathcal{C}_{1} \kappa_{0}+C\left(S T^{3}+h^{2} \delta^{-2}\right), \tilde{M}_{h}^{S+2 \delta, T, \tilde{\kappa}_{n}}\right)
\end{array}
$$

where $\tilde{\kappa}_{n}:=\kappa\left(\tilde{S}_{n}\right)$.

The proof is similar to that of Proposition [3.6. where however Proposition 3.3 and Lemma 3.4 are to be replaced by Proposition 4.5] and Lemma 4.6 respectively. We omit the details.

4.5. Proof of Theorem 1.2. Replacing $h$ by $h / B$ we may assume that $B \equiv 1$. We will show that

$$
\begin{aligned}
h^{1 / 4} N\left(h \Theta_{0}+h^{3 / 2} \mathcal{C}_{1} \kappa_{0}, P_{h}\right)= & \frac{1}{\pi \sqrt{3\left|\xi_{0}\right|}} \int_{0}^{\mathcal{L}}\left(\kappa(s)+\kappa_{0}\right)_{+}^{1 / 2} d s \\
& +\mathcal{O}_{\epsilon}\left(h^{1 / 16-\epsilon}\right)
\end{aligned}
$$

for any $\epsilon>0$. As in Subsection 3.5 we give the proof of the lower bound only. 
Let $N, S$ be as in Subsection 4.4 and let $\tilde{S}_{n} \in\left[s_{n-1}, s_{n}\right]$ be arbitrary with $\tilde{\kappa}_{n}:=\kappa\left(\tilde{S}_{n}\right)$. From Proposition 4.1 and Proposition 4.8 we get for all $\delta \in(0, S / 2], C h^{1 / 2}|\log h| \leq T \leq C^{-1} h^{1 / 4}$ (with $C$ as in Proposition 4.8)

$$
\begin{aligned}
& h^{1 / 4} N\left(h \Theta_{0}+h^{3 / 2} \mathcal{C}_{1} \kappa_{0}, P_{h}\right) \\
& \geq\left(\pi \sqrt{3\left|\xi_{0}\right|}\right)^{-1}(S-\delta) \sum_{n=1}^{N}\left(\tilde{\kappa}_{n}+\kappa_{0}-c_{1}\left(h^{-3 / 2} S T^{3}+h^{1 / 2} \delta^{-2}\right)\right)_{+}^{1 / 2} \\
& \quad-c_{2} N h^{1 / 4} .
\end{aligned}
$$

Now we use the estimate

$$
\begin{aligned}
& \left(\tilde{\kappa}_{n}+\kappa_{0}-c_{1}\left(h^{-3 / 2} S T^{3}+h^{1 / 2} \delta^{-2}\right)\right)_{+}^{1 / 2} \\
& \quad \geq\left(\tilde{\kappa}_{n}+\kappa_{0}\right)_{+}^{1 / 2}-c_{1}^{1 / 2}\left(h^{-3 / 4} S^{1 / 2} T^{3 / 2}+h^{1 / 4} \delta^{-1}\right) .
\end{aligned}
$$

If we assume that $\delta \geq h^{1 / 4}$ we easily deduce from (4.9) that

$$
\begin{aligned}
h^{1 / 4} N\left(h \Theta_{0}+h^{3 / 2} \mathcal{C}_{1} \kappa_{0}, P_{h}\right) \geq( & \left(\sqrt{3\left|\xi_{0}\right|}\right)^{-1} S \sum_{n=1}^{N}\left(\tilde{\kappa}_{n}+\kappa_{0}\right)_{+}^{1 / 2} \\
& \quad-c_{3}\left(h^{-3 / 4} S^{1 / 2} T^{3 / 2}+h^{1 / 4} \delta^{-1}+\delta S^{-1}\right) .
\end{aligned}
$$

Now choose $T=h^{1 / 2-\rho}$ with $0<\rho<1 / 4$. A calculation shows that the second term on the RHS of (4.10) is minimal for the choice

$$
S=h^{1 / 8+3 \rho / 2}, \quad \delta=h^{3 / 16+3 \rho / 4}
$$

and given by $c_{4} h^{1 / 16-3 \rho / 4}$. The first term on the RHS of (4.10) is a Riemannian sum. Recalling that the $\tilde{S}_{n}$ were arbitrary, we finally arrive at the lower bound of (4.8). The upper bound can be established similarly, which concludes the proof of Theorem 1.2 .

\section{REFERENCES}

[Bol] C. Bolley, Familles de branches de bifurcations dans les équations de GinzburgLandau. RAIRO Modél. Math. Anal. Numér. 25 (1991), no. 3, 307-335 (French).

[Bon] V. Bonnaillie, On the fundamental state energy for a Schrödinger operator with magnetic field in domains with corners. Asymptot. Anal. 41 (2005), no. 3-4, 215258.

[CV] Y. Colin de Verdière, L'asymptotique de Weyl pour les bouteilles magnétiques. Comm. Math. Phys. 105 (1986), 327-335 (French).

[DaHe] M. Dauge, B. Helffer, Eigenvalues variation. I. Neumann problem for SturmLiouville operators. J. Differential Equations 104 (1993), no. 2, 243-262.

[DiRa] M. Dimassi, G. Raikov, Spectral asymptotics for quantum Hamiltonians in strong magnetic fields. Cubo Mat. Educ. 3 (2001), no. 2, 317-391.

[FoHe1] S. Fournais, B. Helffer, Accurate eigenvalue estimates for the magnetic Neumann Laplacian. Preprint (2004), to appear in Annales de l'Institut Fourier.

[FoHe2] S. Fournais, B. Helffer, On the third critical field in Ginzburg-Landau theory. Preprint (2005), to appear in Comm. Math. Phys. 
[HeMo] B. Helffer, A. Morame, Magnetic bottles in connection with superconductivity. J. Funct. Anal. 185 (2001), no. 2, 604-680.

[HoSm] K. Hornberger, U. Smilansky, Magnetic edge states. Phys. Rep. 367 (2002), no. 4, 249-385.

[Iv] V. Ivrii, Microlocal analysis and precise spectral asymptotics. Springer Monographs in Mathematics. Springer-Verlag, Berlin, 1998.

[LiSoYn] E. Lieb, J.-P. Solovej, J. Yngvason, Asymptotics of heavy atoms in high magnetic fields. II. Semiclassical regions. Comm. Math. Phys. 161 (1994), no. 1, 77-124.

[LuPa] K. Lu, X.-B. Pan, Estimates of the upper critical field for the Ginzburg-Landau equations of superconductivity. Phys. D 127 (1999), no. 1-2, 73-104.

[Ta] H. Tamura, Asymptotic distribution of eigenvalues for Schrödinger operators with magnetic fields. Nagoya Math. J. 105 (1987), 49-69.

[Tr] F. Truc, Semi-classical asymptotics for magnetic bottles. Asymptot. Anal. 15 (1997), no. 3-4, 385-395.

Royal Institute of Technology, Department of Mathematics, 10044 StockHOLM, SWEDEN

E-mail address: rupert@math.kth.se 